\title{
Spatial Mode Approaches to Enhancing Transport Communications Capacities
}

\author{
T. D Drysdale ${ }^{1}$, B Allen ${ }^{2}$, J Coon $^{2}$ \\ ${ }^{1}$ School of Engineering and Innovation, The Open University, Milton Keynes, MK7 6AA, UK, tim.drysdale@open.ac.uk \\ ${ }^{2}$ School of Engineering Science, The University of Oxford, Parks Rd, OX1 3PJ, UK
}

\begin{abstract}
Transport communications systems face challenges relating to the number, density and velocity of passengers. In the rail environment, it would be advantageous to be able to provide broadband-like data connectivity to all passengers. Due to the limited number of connection points into trackside fibre-optic cables (ca. one per $5 \mathrm{~km}$ ), and the limited spacing possible between trackside-to-train links (ca. $100 \mathrm{~m}$ due to fog), novel solutions for track-side communications backbones are needed. We estimate the total capacity as being in the order of $\mathbf{4 0} \mathbf{G b i t} / \mathrm{s}$, which requires the investigation of novel means of providing spectral efficiency. Spatial modes offer significant benefits, but also bring challenges such as requiring large antenna apertures, which may be tackled by using partial aperture antenna approaches or by employing lenses.
\end{abstract}

Index Terms - antenna, propagation, measurement.

\section{INTRODUCTION}

European countries rely heavily on rail transport, which contributes to $1.1 \%$ of European GDP, yet the share of passenger-km travelled remains below expectations at $6.6 \%$ [1]. In our view, one of the contributing factors to this underutilization is the growing mismatch between the data connectivity available to commuters whilst travelling, compared to at home, work and public-spaces. This limits the ability for medium and long distance travelers to conduct meaningful personal or business activities during their journey, due to the increasing reliance upon peer-to-peer and cloud-based services. For example, by 2020, $48 \%$ of corporate infrastructure spending is expected to accrue to cloud based infrastructure, up from approximately $37 \%$ last year [2].

Existing digital connectivity solutions abound to give enhanced data connectivity in urban areas, where those within buildings benefit from WiFi fed by fibre-optic cables, and mobile users benefit from MIMO and emerging massive MIMO techniques, amongst others. Such approaches exploit either the stationary nature of the buildings (fibre optic cable), or the additional spectral efficiency arising from diversity gain in cluttered environments (MIMO). Yet neither of these solutions is well-suited to the problem of providing broadband-like data capacity to users on the move.

\section{II. DESCRIPTION OF THE CHALLENGE}

While it is obvious that fibre-optic cables cannot be routed to a moving train, the challenges facing the mobile approach to data connectivity on trains relate to:

- density of passengers on a train in terms of data volume and handover requirements

- path loss associated with transmitting through a train wall

-high velocity causing Doppler shift to signals emitted from high-speed trains causing them to cross into, or through, guard-bands allocated in frequency-division multiplexing schemes

- limited diversity gain available outside of urban areas

- line-of-sight limitations imposed by tunnels, cuttings and railway infrastructure.

The number and density of passengers on trains is particularly challenging for mobile networks. We recently estimated the expected data capacity that is needed to handle projected passenger numbers is around $2.5 \mathrm{Gbit} / \mathrm{s}$ per train [3]. In order to estimate a total trackside communications capacity, we next consider that the UK's trackside fibre-optic cable is likely to offer connection points approximately every $5 \mathrm{~km}$, and train spacing as close as $50 \mathrm{~m}$ at slow speeds are theoretically achievable using moving block approaches [4]. This leads us to consider a trackside, bidirectional link capacity requirement of $40 \mathrm{Gbit} / \mathrm{s}$. That link also needs to provide connection points to support the trackside-to-train connectivity. In order to meet the per-train capacity requirements, such a link is likely to use millimetre-wave spectrum and beam steering techniques. Note that at $60 \mathrm{GHz}$, in the UK, fog limits the maximum link distance [5] to perhaps $100 \mathrm{~m}$ or less, so as to ensure $99.999 \%$ availability [3]. However, this is likely to be relaxed for lower frequency bands in terms of fog, but limitations on equipment capability and spectrum regulation would likely point to link distances in the same order at that for $60 \mathrm{GHz}$.

Thus, the need for regular trackside connection points suggests that a relay network will be required, particularly in light of the speed of handover between trackside connection points as trains make their journey. Such relay networks are often found in wireless systems, and so an opportunity arises to consider approaches that avoid the cost and infrastructure constraints associated with trenching cables, i.e. a wireless relay backbone. 


\section{CONCEPT OF SPATIAL Mode WiRELESS Relay NETWORKS}

The limited availability of electromagnetic spectrum suggests that a $40 \mathrm{Gbit} / \mathrm{s}$ wireless relay network will require a significant enhancement of its spectral efficiency. It is well known that, under the constraint of constant power, that a substantial increase in total capacity can be achieved by splitting the available power across multiple channels. The relative capacity increase for a given fixed bandwidth can be calculated as follows. We start with the well-known Shannon-Hartley formula for the capacity of a single channel, one hop link, originally derived by Shannon [6]:

$$
C=B \log _{2}\left(1+\frac{S}{N}\right),
$$

where $C$ is the channel capacity in bit/s, $B$ is the channel bandwidth in $\mathrm{Hz}$, and average white Gaussian noise is assumed with a signal to noise ratio of $S / N$. If the same power is split across $n$ channels, then the total capacity becomes:

$$
C_{n}=n B \log _{2}\left(1+\frac{S / n}{N}\right) .
$$

The relative increase in the total capacity can be found by taking the ratio of Eq.(2) to Eq. (1):

$$
\frac{C_{n}}{C}=\frac{n \cdot \log _{2}\left(1+\frac{S / n}{N}\right)}{\log _{2}\left(1+\frac{S}{N}\right)} .
$$

For the sake of illustration, we show in Table 1 the value of $C_{n} / C$ for channel counts of up to 13 .

TABLE I. CAPACITY INCREASE IN MULTICHANNEL SYSTEMS

\begin{tabular}{|c|c|}
\hline Number of channels $(\boldsymbol{n})$ & Relative Capacity $\left(\mathbf{C}_{\mathbf{n}} / \mathbf{C}\right)$ \\
\hline 1 & 1.0 \\
\hline 3 & 2.1 \\
\hline 5 & 2.9 \\
\hline 7 & 3.4 \\
\hline 9 & 3.9 \\
\hline 11 & 4.3 \\
\hline 13 & 4.6 \\
\hline
\end{tabular}

In the case of millimeter-wave systems, the limited output power of existing solid-state local oscillators suggests the adoption of a multi-channel approach, but there are diminishing returns to adding many channels and a price to pay in complexity. For a $40 \mathrm{Gbit} / \mathrm{s}$ capacity link, assuming $6 \mathrm{GHz}$ of bandwidth, and sufficient power to provide $30 \mathrm{~dB}$ SNR on a single-channel link, then Equation (1) indicates 9 channels are required. Once the power is split between the nine channels, the individual signal to noise ratios are on the order of $11 \mathrm{~dB}$, indicating that QPSK modulation would be preferable [7]. However, a full analysis of the transmit power and related modulation scheme choices is outside the scope of this paper. The use of polarisation states enables two channels to be provided, but assuming limited or nonexistent diversity gain (i.e. no clutter, e.g. due to a rural environment), then an alternative approach must also be considered so as to provide additional channels.

One such approach is to consider the generation and reception of spatial radio modes, such as orbital angular momentum [8]. Originally developed for optical bands [9], the additional channels provided by spatial modes result from the generation of spatial phase profiles that are capable of being independently detected, so long as the receiver aperture is in some way distributed such that it can detect and discriminate the prevailing phase profile. They are not necessarily challenging to produce, with simple switching schemes being sufficient in some applications [10]. An illustration of what a trackside link exploiting spatial modes could look like is in Figure 1.

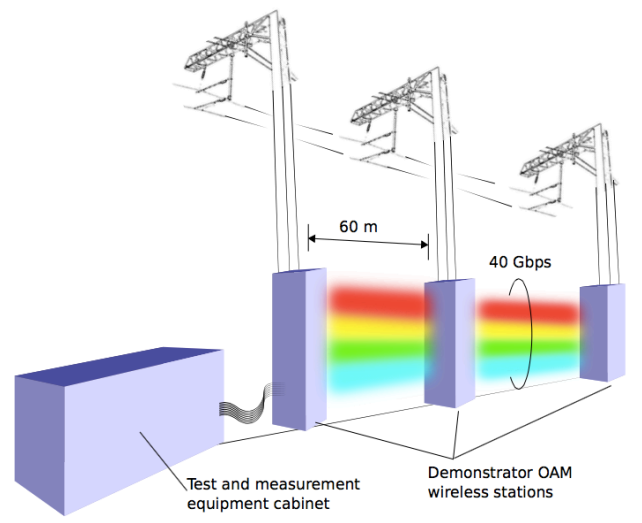

Fig. 1. Illustration of a possible spatial-mode relay network demonstration system showing a two hop link mounted on overhead electrification gantries [9]

\section{IMPLEMENTATION CHALLENGES}

A regular criticism of spatial mode schemes is that they require large antenna apertures and place severe limits on the link distance, and even appear to be equivalent to a near-field effect [11]. However, the modes are indeed propagating as shown experimentally in [12-13], but have a null on the boresight that results in a fourth order reduction in the power with distance unless the receiver aperture is increased to match the mode [14]. There are two routes to ameliorating this aspect of spatial modes. One is to design transmit antennas to produce smaller nulls, for example by using lenses; and the other is to develop receiver architectures that permit smaller apertures to receive the modes correctly. The rest of the paper concerns the latter approach.

The partial aperture receiver requires the arrangement of receive antennas to suit the combination of incoming OAM modes, such that they maintain their independence [8]. Such an approach has been shown to work experimentally, giving 
crosstalk between channels below $-11 \mathrm{~dB}$ [15] in the case of a four-mode system (with orbital angular momentum mode numbers of $+/-1,+/-2$ ). The experimental apparatus used to test this concept is shown in Fig. 2 and consists of an 8element circular transmit array of monopoles; power amplifier; digitally programmable phase shifters; and partial aperture receive array with programmable phase shifters. The equipment was chosen to operate in the $5 \mathrm{GHz}$ band.

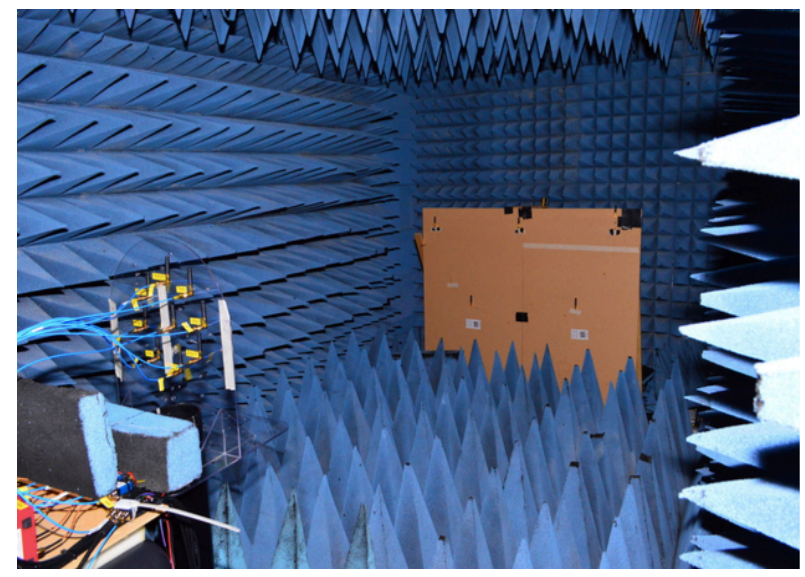

Fig. 2. Photograph of the experimental setup for system performance measurements [15]

\section{CONCLUSIONS}

Since transport corridors have low diversity gain, but there is growing demand for broadband-like data connectivity, alternative communications backbone technologies are needed. We are exploring the use of spatial modes to compensate for the low diversity gain. Challenges include the requirement for a large size of the receive antenna, given the boresight null of orbital angular momentum modes. We have approached this implementation challenge by designed a receive antenna that does not need to sample the whole area of the mode in order to maintain independence between the channels. In work to date, the measured crosstalk between channels is better than $-11 \mathrm{~dB}$ for four spatial modes, with further improvements to be expected.

\section{ACKNOWLEDGMENT}

T. D. Drysdale thanks The Department of Transport for funding under the T-TRIG scheme, and H.M. Govt. for their support.

\section{REFERENCES}

[1] Report by Steer Davies Gleave (London) for Directorate General for Mobility and Transport (Rue de Mot 28 B-1040, Brussels, Belgium), "Study on the Cost and Contribution of the Rail Sector European Commission (MOVE/B2/2014-761), September 2015.

[2] International Data Corporation, "Worldwide Quarterly Cloud IT Infrastructure Tracker," $11 \quad$ April 2016. https://www.idc.com/getdoc.jsp?containerId=prUS41176816 Last accessed: 25 October 2017

[3] T. D. Drysdale, B. Allen, and J. P. Coon. "Enhanced spectral efficiency of wireless communications alongside transport infrastructure.” In IET Antennas, Propagation \& RF Technology for Transport and Autonomous Platforms. February 2017.
[4] L. V. Pearson, "Moving Block Railway Signalling," PhD Thesis, Loughborough University of Technology, 1973

[5] S.Ventouras, S.A. Callaghan, I.Clark, A.Burges, G.Porter, J.R.Norbury, S.M. Feeny, S. Salous, "Radio Systems at $60 \mathrm{GHz}$ and Above," Report on Ofcom Contract 410000258, 2006

[6] C. E. Shannon, "A mathematical theory of communication," Bell Systems Technical Journal Vol. 23, p623-656, 1948

[7] T. L. Alumona, G.N Onoh, P. C. Mbanugo , O.O. Ugwuoke O.O, "Signal to noise ratio estimation of QAM and QPSK Modulation technique at $910 \mathrm{MHz}$ and $2116.4 \mathrm{MHz}$ Using measured data," International Journal of Advanced Research in Computer Engineering \& Technology (IJARCET), Volume 3 Issue 10, October 2014

[8] C. J. Vourch, B. Allen, T. D. Drysdale, "Planar millimetre-wave antenna simultaneously producing four orbital angular momentum modes and associated multi-element receiver array," IET Microwave Antennas and Propagation, vol. 10, no. 14, pp. 1492-1499, 2016

[9] L. Allen, M. W. Beijersbergen, R. J. C. Spreeuw, and J. P. Woerdman, "Orbital angular momentum of light and the transformation of Laguerre-Gaussian laser modes," Phys. Rev. A, Vol. 45, No. 11, June 1992

[10] A. Tennant and B. Allen, "Generation of OAM radio waves using circular time-switched array antenna," in Electronics Letters, vol. 48, no. 21, pp. 1365-1366, October 112012

[11] O. Edfors and A. J. Johansson, "Is Orbital Angular Momentum (OAM) Based Radio Communication an Unexploited Area?," in IEEE Transactions on Antennas and Propagation, vol. 60, no. 2, pp. 1126-1131, Feb. 2012

[12] Y. Yan, G. Xie, M. P. J. Lavery, et al. "High-capacity millimetrewave communications with orbital angular momentum multiplexing,"Nat. Comms., 5, 4876, March 2014

[13] W Zhang, S Zheng, X Hui, R Dong, X Jin, H Chi, X Zhang, "Mode Division Multiplexing CommunicATIONS Using Microwave Orbital Angular Momentum", IEEE Trans. Wireless Communications, Vol. 16, No. 2, Feb. 2017

[14] T. D. Drysdale, B. Allen, E. Cano, Q. Bai, and A. Tennant. "Evaluation of OAM-radio mode detection using the phase gradient method." In th European Conference on Antennas and Propagation (EuCAP). March .

[15] T. D. Drysdale, "Multiple Antenna Line-of-Sight Test Bed," Final Report to the Department of Transport, March 2017. 